\title{
Functional crystalline materials based on macrocyclic nanochannels
}

\author{
S. Sato \\ Department of Applied Chemistry, The University of Tokyo, 7-3-1 Hongo, Bunkyo-ku, Tokyo 113-8656 \\ satosota@appchem.t.u-tokyo.ac.jp
}

\begin{abstract}
Nanocarbon materials show attractive functions in device applications, however the structural deviations and ambiguity disturb understanding between the functions and the structures. We have worked on the synthesis of molecular nanocarbon materials based on a simple strategy of macrocyclization of aromatic units. The molecular structures and supramolecular integrated structures can be fully accessed at the precision of molecular level, and unparalleled functions derived from the unique structures were found.
\end{abstract}

For example, a macrocyclic hydrocarbon molecule, [6]cyclo-2,7-naphthylene ([6]CNAP), synthesized by single bond linkage of six naphthylene units (Figure 1a) has a cyclic structure equivalent to an atom-defective structure of graphene [1]. In this study, [6]CNAP was applied to a negative electrode active material for a rechargeable lithium battery, where graphite is conventionally and commercially used as the material. All-solid-state lithium battery with $\mathrm{LiBH}_{4}$ as electrolyte was constructed with three layers simply by uni-directional pressing: the composite electrode with [6]CNAP, acetylene black (AB) and $\mathrm{LiBH}_{4}\left|\mathrm{LiBH}_{4}\right| \mathrm{Li}$ (Figure 1b and c). Depending on purification methods, the recyclability of the rechargeable batteries largely differed. Surprisingly, highly purified specimen by sublimation method showed poor recyclability, and the recrystallized specimen from organic solvents showed stable recyclability up to 65 discharge-charge cycles and around twice battery capacity than a graphite electrode. We found that the differences in battery performance were originated from the molecular packing structures in solid states by powder X-ray structural analyses with Rietveld refinement. The key for the high battery performance is the one-dimensional nanopores constructed from the assembly of the central pore of [6]CNAP and $\pi$-stacks of naphthylene units. The quantitative battery performance results and the precisely determined packing structures showed that lithium ion is stored by the intercalation between naphthylene units and also in the one-dimensional nanopores to afford the high battery capacity. We successfully revealed the relationship between unique packing structures and battery performance [2].

(a)

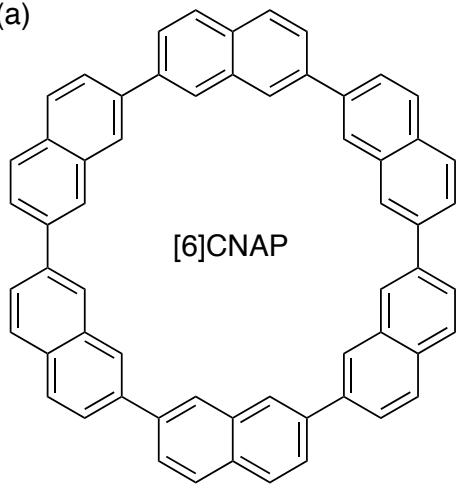

(b)

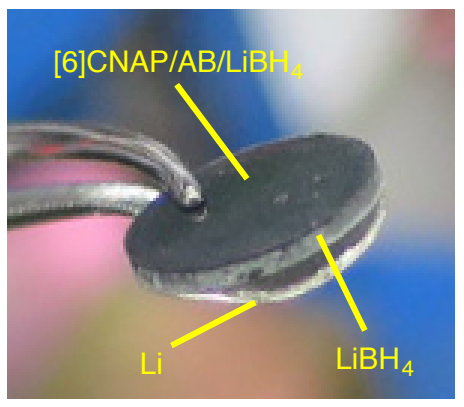

(c)

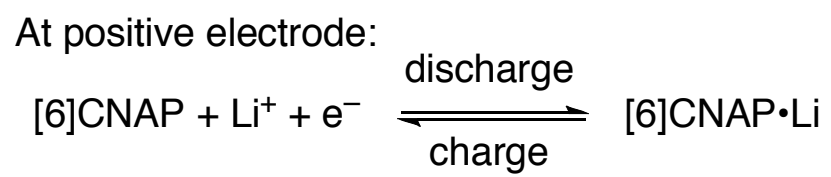

At negative electrode:

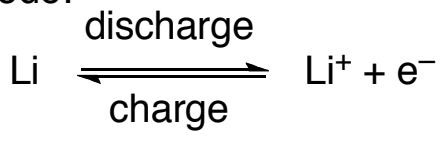

Figure 1. (a) Chemical structure of [6]CNAP. (b) The photo of bulk-type all-solid-state lithium battery. Cell diameter is 8 mm. (c) Redox reactions at the electrodes.

[1] Nakanishi, W., Yoshioka, T., Taka, H., Xue, J. Y., Kita, H. \& Isobe, H. (2011). Angew. Chem. Int. Ed. $50,5323$.

[2] Sato, S., Unemoto, A., Ikeda, T., Orimo, S. \& Isobe, H. (2016). Small 12, 3381.

Keywords: aromatic macrocycle; nanochannel; powder diffraction; single-crystal diffraction; lithium ion battery

The author thanks K. Sato, H. Ohmiya, and N. Warifune (IMR) for technical assistance with battery fabrication, M. Oinuma, T. Saitou, and H. Seki (ERATO) for assistance with synthesis and SPring-8 (2015A1312, 2015B1499) for the use of the X-ray diffraction instruments. This study was partly supported by ERATO Program of JST (JPMJER1301), Target Project 4 of AIMR, Integrated Materials Research Center for the Low-Carbon Society (LC-IMR), ALCA Program of JST, and KAKENHI (24241036, 25102007, 25220911). 